\title{
Correlation of COX- 2 Expression in Colorectal Carcinoma with Clinicopathological Features
}

\author{
Sandhya VENKATACHALA ${ }^{1}$, Manjula RAJENDRAN ${ }^{2}$ \\ Department of Pathology, 'Apollo Hospitals, BANGALORE, INDIA and ${ }^{2}$ Government Sivagangai Medical College \& Hospital, SIVAGANGAI, INDIA
}

\begin{abstract}
Objective: Colorectal carcinoma is the most common neoplasm of the gastrointestinal tract. COX-2 expression is upregulated in colorectal carcinoma. Therefore its assessment would identify patients amenable to adjuvant anticyclooxygenase therapy. We studied COX-2 immunoexpression in colorectal adenocarcinoma and correlated it with clinicopathological features as age, gender, tumor location, tumor size, degree of differentiation, and TNM stage.

Material and Method: Sixty-five consecutive cases of colorectal adenocarcinoma were retrieved from the records of the Pathology Department of a tertiary care hospital. The tumors were categorized as low positive and high positive based on their total COX-2 scores, which is the sum of proportion and intensity scores of immunostaining, and were correlated with clinicopathological features. Statistical analysis was done using the Chi-square test and Kendall's Tau-b correlation method.

Results: COX-2 was expressed in $86.2 \%$ of cases including $90 \%$ of left colonic carcinomas and $77.3 \%$ of right colonic carcinomas. Lymph node metastasis was present in $22.2 \%, 25 \%$ and $47.75 \%$ of COX-2 negative, low positive and high positive tumors respectively. High positive COX-2 cases constituted $33.3 \%$ of stage I, $58.8 \%$ of stage II, $80 \%$ of stage III and $100 \%$ of stage IV tumors. About $56.6 \%$ of well differentiated, $66.6 \%$ of moderately differentiated and $100 \%$ of poorly differentiated carcinomas showed high COX-2 expression. The COX-2 overexpression was associated with advancing depth of invasion $(\mathrm{p}=0.021)$, stage of tumor $(\mathrm{p}=0.05)$, more frequent lymph node metastasis and decreasing degree of differentiation.
\end{abstract}

Conclusion: The association of COX-2 overexpression with increasing stage and depth of invasion may justify the use of COX-2 inhibitors as an adjuvant to chemo and radiotherapy.

Key Words: Adenocarcinoma, Colorectal neoplasms, Cyclooxygenase 2, Cyclooxygenase 2 inhibitors, Lymphatic metastasis.

\section{INTRODUCTION}

Colorectal carcinoma is the fourth common cancer in the world and second most common cause of cancer-related death (1). Epidemiological studies data have shown a lower incidence of adenomas and carcinomas in patients taking Non Steroidal Anti-Inflammatory Drugs (NSAIDs) for a long time suggesting a pathogenic role for cyclooxygenase (COX-2) in colonic tumorigenesis (2-4). COX-2, an inducible isoform of cyclooxygenase is usually absent or present in low levels in normal colonic epithelium and is upregulated in colorectal carcinoma (5). Assessment of this molecular factor would therefore help in identifying the patients who are likely to benefit from COX-2 inhibitor adjuvant therapy which attenuates the metastatic potential of colorectal carcinoma, thereby improving the prognosis. Therefore the present study aims to evaluate COX-2 expression in colorectal carcinoma and to correlate it with clinopathological features as age, sex, tumor location, size, depth of invasion, histological type, degree of differentiation, lymph node metastasis and stage of the tumor.

(Turk Patoloji Derg 2017, 33:228-234)

Received : 27.06.2016 Accepted : 13.12.2016

\section{MATERIAL and METHODS}

Sixty-five consecutive resection specimens of colorectal carcinoma diagnosed between January 2009 and December 2013 were retrieved from the records of the pathology department at a tertiary care hospital. Hematoxylin and eosin slides of the 65 cases were reevaluated in respect to the location, histological type and grade of the neoplasm, depth of invasion and lymph node metastasis. Clinical details which included the age, gender, stage of tumor were recorded. The representative slides were selected in each case that included primary tumor and, lymph nodes or satellite nodules. If the case had distant metastasis, the metastatic deposits were also included. Immunohistochemical staining for COX-2 was done by the following procedure (6). Four microns thick sections were taken, floated on to Poly-L-Lysine coated slides. Deparaffinization followed by dexylinization and rehydration was done. Antigen retrieval was done by pressure cooking in citrate buffer $(\mathrm{pH}-6.0)$ for 10 minutes. After peroxidase block, the sections were covered with concerned primary antibody (Monoclonal

Correspondence: Sandhya VENKATACHALA

Department of Pathology, Apollo Hospitals, BANGALORE, INDIA

E-mail: sandhyavenkatachala@yahoo.co.in Phone: +9591627364 
Rabbit Anti-Human COX-2, THERMO Scientific) for 1 hour. Super enhancer was then added and left for 30 minutes. It was washed with TBS buffer 3 times. Super sensitive Poly- HRP was added. The slides were treated with colour development solution 3'3' diaminobenzidine (DAB) for 5-8 minutes. Counterstaining was done with haematoxylin stain. The slides were air dried, cleared in xylene and mounted with DPX.

A section of lung adenocarcinoma was used as positive control and the negative control was the same tissue incubated without secondary antibody. COX-2 expression was evaluated using a method wherein both the percentage of positive cells (proportion score) and the intensity of staining (intensity score) were added to obtain the "total COX-2 score" $(7,8)$. The proportion score was scaled between 0 and 4 (0: No cells are positive, 1: $1 \%$ to $25 \%$ cells are positive, $2: 26 \%$ to $50 \%$ cells are positive, $3: 51 \%$ to $75 \%$ cells are positive and $4: 76 \%$ to $100 \%$ cells are positive). The intensity score was scaled between 0 and 3 (0: No staining, 1: Weak staining, 2: Intermediate staining, 3: Strong staining). The stratification of staining intensity was made in comparison with the staining intensity of inflammatory cells. When the staining intensity of tumor cells was less than the inflammatory cells, it was considered weak staining (intensity score-1). Similarly when tumor cell staining was the same and stronger than inflammatory cells, it was considered intermediate (score 2) and strong staining (score 3), respectively (Figure 1A-D). "Total COX-2 score" thus obtained was used to categorize COX-2 expression of tumors as "Negative (total score 0 to 2)", "Low positive (total score 3 to 4 )" and "High positive (total score 5 to 7 )".

COX-2 expression (total COX-2 score) was then correlated with clinicopathological features using the statistical methods of Chi-square test and Kendall $(\tau)$ Tau-b tests.
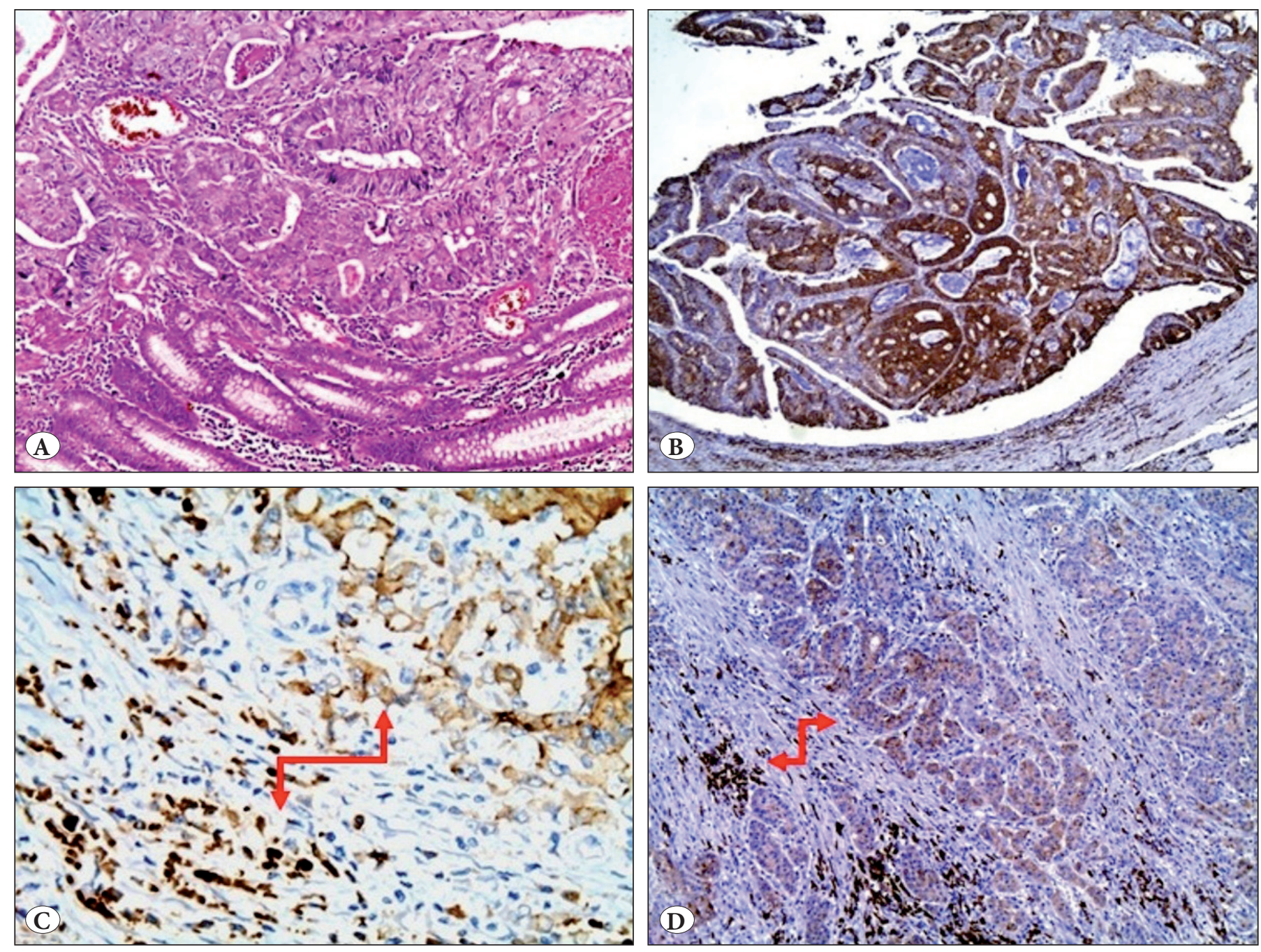

Figure 1: A) Well-differentiated adenocarcinoma (H\&E; x100). B) Strong COX-2 expression (COX-2; x100). C) Moderate COX-2 staining (COX-2; x400). D) Weak COX-2 expression (COX-2; x100) (Note the red arrow in C\&D to compare with inflammatory cells). 
The Chi-square test was used for determining correlation between COX-2 expression and pathological features such as depth of tumor, microscopic grade, lymph node metastasis, and TNM stage. Relationship between age, gender and COX-2 expression was determined by Kendall $\tau$-b correlation.

\section{RESULTS}

Of the 65 cases of colorectal carcinoma included in the study, $56(86.2 \%)$ cases expressed COX-2 while 9 cases (13.8\%) were COX-2 negative. Forty-four (67.7\%) of the 56 positive cases, strongly expressed COX-2 while 12 (18.5\%) weakly expressed COX-2. Mean age of presentation was 64 years-old \pm Standard Deviation (Range: 38 years-93 years,) with males (36 cases) slightly outnumbering the females (29 cases). Forty cases $(61 \%)$ were located in left colon, while $22(34 \%)$ and three cases (4\%) were located in right colon and transverse colon, respectively. Ninety percent of left colonic carcinomas and $77.3 \%$ of right colonic carcinomas expressed COX-2. Three stage I, 34 stage II, 25 stage III and three stage IV tumors were seen in the present study with one pT1, three pT2, 48 pT3 and 13 pT4 tumors. Lymph node metastasis was seen in 26 of the 65 cases (40\%) with $15 \mathrm{pN} 1$ and $11 \mathrm{pN} 2$ cases. Two of the three stage IV cases showed metastatic deposits in both ovaries while one showed deposits in uterus and fallopian tube.

\section{COX-2 Negative Cases}

Four of the nine COX-2 negative cases did not show any expression of COX-2. However in the remaining five cases, less than $25 \%$ of tumor cells weakly expressed COX2 accounting to a total score of 2 which was considered negative. Five were located in the right colon while four were in the left. No lymph node metastasis was seen in 7 of the nine (77.8\%) COX-2 negative cases. Seven stage II and two stage III tumors were seen. Only 2 cases (22.2\%) had tumor in the lymph node and the tumor cells in one of them were low positive for COX-2.

\section{COX-2 Low Positive Cases}

Of the twelve low positive tumors, three were located in the right and nine in the left colon. Two stage I, seven stage II, three Stage III tumors were seen. There was no stage IV tumor. Nine cases $(75 \%)$ had no evidence of tumor in the lymph node. In two of three cases $(25 \%)$ with lymph node deposits, a similar COX-2 expression was seen in both the primary tumor and the lymph node deposits while a higher expression was seen in the lymph node deposits in the third case in the N2 stage. The morphology of primary tumor and the metastasis was however the same.

\section{COX-2 High Positive Cases}

The location of these 44 tumors was 27 (61.4\%) in the left colon, $14(31.8 \%)$ in the right colon, and three in the transverse colon. COX-2 high positive tumors included one stage I, 20 stage II, 20 stage III and three stage IV tumors. Lymph node deposits were seen in 21 cases $(47.7 \%)$ of which 11 were in $\mathrm{N} 1$ stage and 10 were in N2 stage. Twenty-three cases $(52.3 \%)$ did not have lymph node metastasis. One (2.2\%) T2 tumor, 34 (77.3\%) T3 tumors and 9 (20.5\%) T4 tumors were present in this group. There was no $\mathrm{T} 1$ tumor. Stage I, stage II, stage III and stage IV tumors constituted one case (2.2\%), 20 cases (45.5\%), 20 cases $(45.5 \%)$ and three cases $(6.8 \%)$ respectively. The organs involved by distant metastasis in the three stage IV tumors were the fallopian tube and ovary in two of them and an umbilical nodule in the third. All of the mucinous and signet ring cell carcinomas showed high COX-2 positivity (Figure 2A,B and $3 \mathrm{~A}, \mathrm{~B})$.
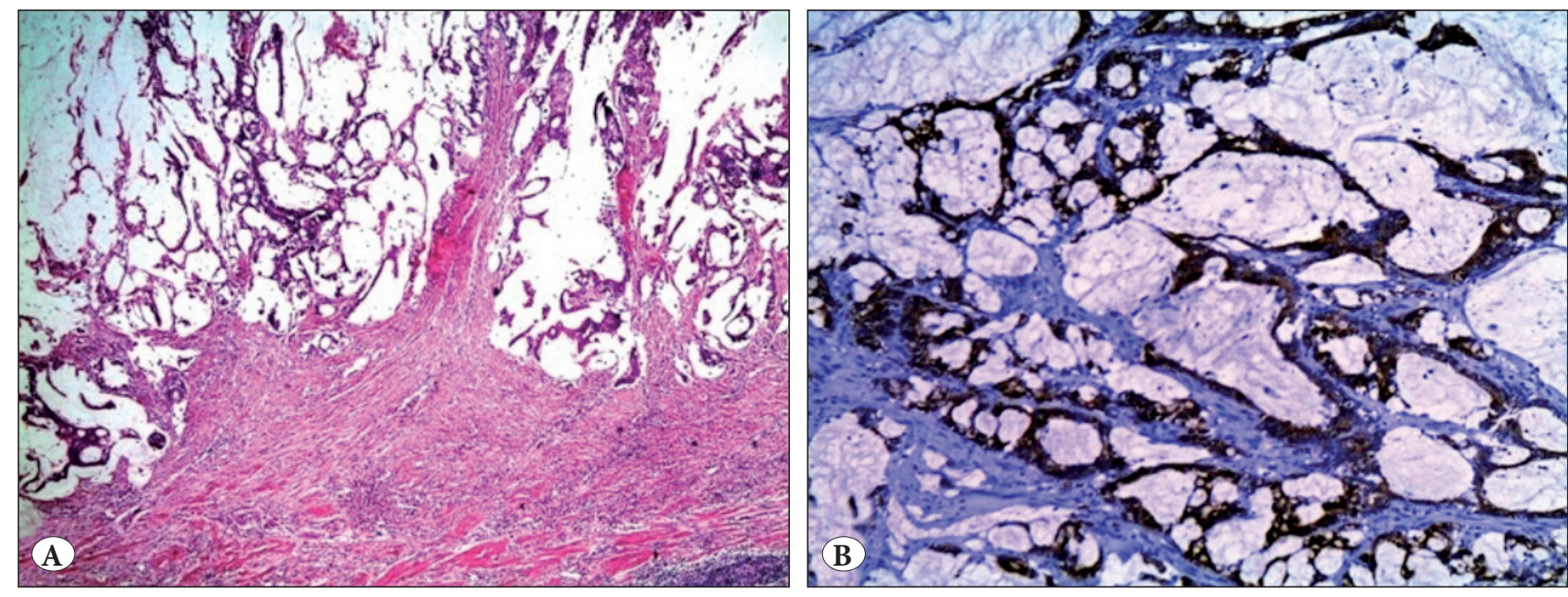

Figure 2: A) Mucinous carcinoma with extracellular mucin (H\&E; x100). B) Strong COX-2 expression in the same tumor (COX-2; x400). 
Lymph node metastasis was present in $22.2 \%, 25 \%$ and $47.8 \%$ of COX-2 negative, low positive and high positive cases, respectively (Figure 4A,B). This difference however was not statistically significant $(\mathrm{p}$ value $=0.08$ )

The distribution of cases and correlation between COX-2 expression, stage, the depth of invasion, histopathological grade and tumor type have been shown in Tables I-III.

\section{COX-2 Expression and Location of Tumor}

Among 65 cases 22 cases (33.9\%) were located in right colon, 40 cases $(61.5 \%)$ were located in of left colon and three cases $(4.6 \%)$ were located in the transverse colon. Of the 22 right colonic carcinomas, 17 (77.3\%) expressed COX-2 and 5 (22.7\%) were negative. The forty cases of left colonic carcinoma included 4 (10\%) COX-2 negative tumors and $36(90 \%)$ COX-2 positive tumors.

\section{COX-2 Expression and Size of Tumor}

The greatest diameter of the nine COX-2 negative tumors varied between $2.5 \mathrm{~cm}$ and $6.0 \mathrm{~cm}$ (mean $4.6 \mathrm{~cm} \pm \mathrm{SD}$ ) while those of 56 COX-2 positive tumors ranged from 2.5 $\mathrm{cm}$ to $12.0 \mathrm{~cm}$ (mean $5.3 \mathrm{~cm} \pm \mathrm{SD}$ ). The difference was not statistically significant $(\mathrm{p}=0.27)$.
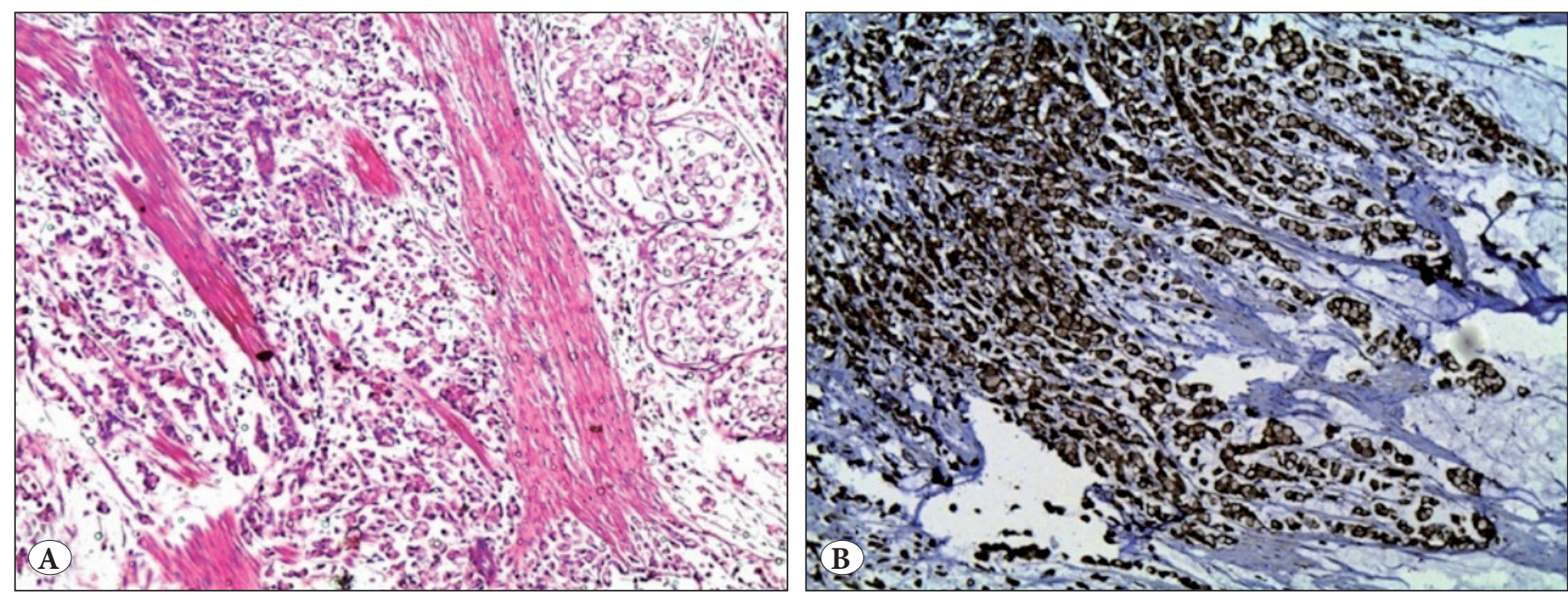

Figure 3: A) Signet ring cell carcinoma infiltrating the muscle layer (H\&E; x100). B) Strong COX-2 expression in signet ring cells (COX$2 ; \mathrm{x} 100)$.
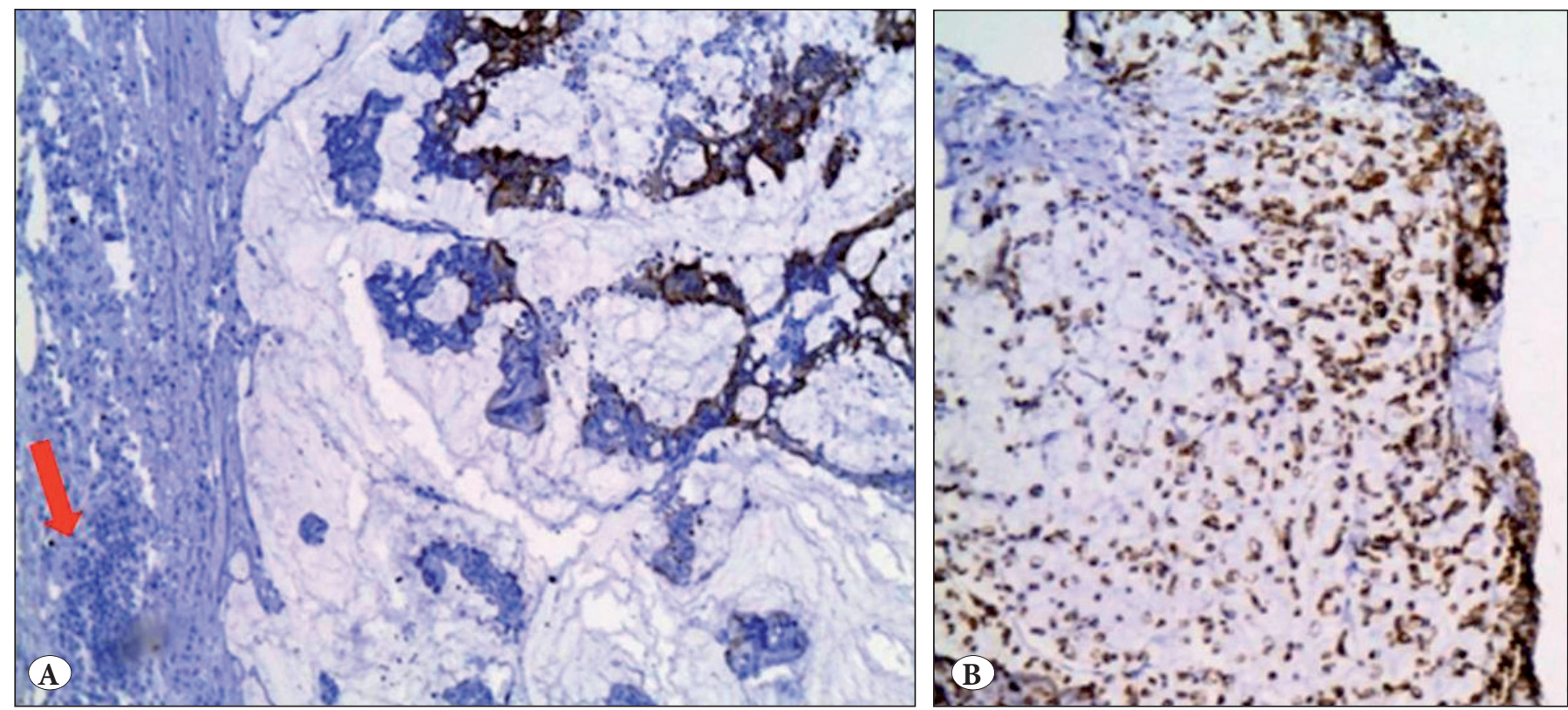

Figure 4: A) Strong COX-2 staining in 30\% of metastatic tumor cells in lymph node. Red arrow shows capsule with few lymphoid cells (COX-2; $\mathrm{x} 100)$. B) Peritoneal nodule with COX-2 expressing signet ring cells (COX-2; x100). 
Table I: The relation between COX-2 expression and the stage

\begin{tabular}{lcccc}
\hline \multirow{2}{*}{ Stage of tumor* } & Total number of cases (n, \%) & \multirow{2}{*}{ Negative (n, \%) } & \multicolumn{2}{c}{ POX-2 expression } \\
\cline { 3 - 5 } & & Low positive & High positive \\
\hline Stage I & $3(4,6 \%)$ & Nil $(0 \%)$ & $2(66.7 \%)$ & $1(33.3 \%)$ \\
\hline Stage II & $34(52.3 \%)$ & $2(20.6 \%)$ & $7(20.6 \%)$ & $20(58.8 \%)$ \\
\hline Stage III) & $25(38.4 \%)$ & Nil $(0 \%)$ & Nil $(0 \%)$ & $3(10 \%)$ \\
\hline Stage IV $)$ & $3(4.6 \%)$ & $9(13.9 \%)$ & $12(18.4 \%)$ & $44(67.7 \%)$ \\
\hline Total & $65(100 \%)$ & & &
\end{tabular}

${ }^{*} \mathrm{p}$ value is 0.05

Table II: The relation between COX-2 expression and depth of invasion

\begin{tabular}{lcccc}
\hline \multirow{2}{*}{ Depth of invasion $^{*}$} & Total number of cases (n, \%) & \multirow{2}{*}{ Negative } & \multicolumn{2}{c}{ COX-2 expression } \\
\cline { 3 - 5 } & & & \multicolumn{2}{c}{ Positive } \\
\cline { 3 - 5 } & $1(1.5 \%)$ & Nil $(0)$ & Nil $(0 \%)$ & Nil $(0 \%)$ \\
\hline T1 & $3(4.6 \%)$ & $5(10.4 \%)$ & $10(20.8 \%)$ & $33(68.8 \%)$ \\
\hline T2 & $48(73.8 \%)$ & $3(23 \%)$ & Nil $(0 \%)$ & $10(77 \%)$ \\
\hline T3 & $13(20 \%)$ & $9(13.9 \%)$ & $12(18.4 \%)$ & $44(67.7 \%)$ \\
\hline T4 & $65(100 \%)$ & & & \\
\hline
\end{tabular}

${ }^{*} \mathrm{p}$ value is 0.021

Table III: COX-2 expression in the different histological types and grade of colorectal carcinoma

\begin{tabular}{lcccc}
\hline \multirow{2}{*}{ Histological grade and type $^{*}$} & \multicolumn{3}{c}{ COX-2 expression } \\
\cline { 2 - 5 } & Total number of cases (n, \%) & Negative & Low positive & High positive \\
\hline Well differentiated adenocarcinoma & $16(24.6 \%)$ & $3(18.8 \%)$ & $4(25 \%)$ & $9(56.2 \%)$ \\
\hline Moderately differentiated adenocarcinoma & $42(64.6 \%)$ & $6(14.3 \%)$ & $8(19 \%)$ & $28(66.6 \%)$ \\
\hline Poorly differentiated adenocarcinoma & $1(1.5 \%)$ & 0 & 0 & $1(100 \%)$ \\
\hline Mucinous adenocarcinoma & $5(7.7 \%)$ & 0 & 0 & $5(100 \%)$ \\
\hline Signet ring cell adenocarcinoma & $1(1.5 \%)$ & 0 & 0 & $1(100 \%)$ \\
\hline
\end{tabular}

${ }^{* * *} \mathrm{p}$ value is 0.9 .

\section{COX-2 Expression and Stage of Tumor}

The correlation between depth of invasion and COX-2 expression was statistically significant $(\mathrm{p}=0.021)$. The stages of tumors also bear a significant correlation with COX-2 expression, $p$ value being equal to 0.05 . The $p$ values for correlation with lymph node metastasis were 0.08 .

\section{COX-2 Expression and Other Clinicopathological Features}

The $\mathrm{p}$ values for correlation with age, gender, and microscopic grade were $0.82 ; 0.47$; and 0.08 respectively.

\section{DISCUSSION}

Colorectal cancer is the fourth most common cancer in the world and the second most common cause of cancer related death (1). Assessment of molecular prognostic factors would be of great help for identifying patients who would benefit from adjuvant therapies, thereby increasing their survival. Cyclooxygenase-2 (COX-2) and its product $\mathrm{PGE}_{2}$ play an important role at different levels in colorectal carcinogenesis. It has been implicated from the initiation stage to tumor progression (9). Some of its carcinogenetic mechanisms include evasion of apoptosis, increasing bcl2 expression and inhibition of Fas-mediated apoptosis, activation of the APC- $\beta$ catenin pathway, increasing the expression of vascular endothelial growth factor (VEGF), decreasing E-cadherin expression favoring epithelialmesenchymal transition (EMT) and increasing matrix metalloproteinases-2 (MMP-2) thereby enhancing the invasiveness and metastatic potential of the tumor cells (10). 
The present study was thus done to analyse the relation between COX-2 expression and the biological characteristics of colorectal carcinoma.

In the present study of the 65 colorectal carcinomas, $86.2 \%$ of cases expressed COX-2 while $13.8 \%$ did not express. Similar expression has been seen by Al-Maghrabi who found $85 \%$ of colorectal carcinomas expressing COX-2 (11). However other studies as published by Soumaoro et al. have shown relatively fewer tumors (70\%) expressing COX-2 (7).

The present study included fifty-nine (90.6\%) stage II/ III tumors with only three (4.6\%) of the stage I tumors contributing to the high percentage of COX-2 expressing tumors. The difference noted in studies by Al Maghrabi and Soumaro could be attributed to the difference in location of the tumors in their series. In the former study by $\mathrm{Al} \mathrm{Maghrabi,} \mathrm{similar} \mathrm{to} \mathrm{our} \mathrm{study} \mathrm{greater} \mathrm{numbers} \mathrm{of}$ left colon carcinoma were present, while in the latter a greater number of right colon carcinoma were included. Right colon carcinomas are more frequently associated with microsatellite instability and lower or absent COX2 expression while left colon carcinomas are frequently associated with loss of APC and hence increased COX-2 expression mediated by $\beta$ catenin $(12,13)$. In the absence of APC, $\beta$ catenin translocates to the nucleus and increases COX-2 levels. Nasir et al. and others found an inverse correlation between COX-2 and APC expression and a more frequent COX-2 expression in left colonic carcinoma (13). In agreement with these findings, $90 \%$ of left colon carcinomas were COX-2 positive while only $77.3 \%$ of right colon carcinomas expressed COX-2 in the present study.

Lymph node metastasis was present in $22.2 \%, 25 \%$ and $47.8 \%$ of COX-2 negative, low positive and high positive cases respectively. Though the values were not statistically significant, these findings are in consistence with those of Sheehan KM (12) and Al-Maghrabi (11) who also found two to four times more frequent lymph node metastasis in tumors with high COX-2 expression. Furthermore, in 76\% of stage III tumors with lymph node metastasis, a similar staining intensity was seen in the primary tumor and lymph node deposits, while in $16 \%$ of the cases, the primary tumor was high positive, whilst the lymph node deposits being low positive. However, no larger series are available to compare these findings. With increasing stage of the tumor, there was an increase in number of high COX-2 positive tumors from $33.3 \%, 58.8 \%, 80 \%$ to $100 \%$ in stage I, II, III, IV tumors respectively. On the contrary the number of low COX-2 expressing tumors gradually declined as the stages increased and there was no COX-2 negative carcinoma in stage IV. These findings are in correlation with other studies that used the Dukes classification $(12,14)$ or the AJCC staging systems $(11,15)$. Elzagheid et al. have also found high COX-2 expression in association with advanced stages of colorectal carcinoma while finding no significant association with tumor grade and lymph node status (16). As the tumor depth of invasion progressed from $\mathrm{T} 1$ to $\mathrm{T} 4$, invading the submucosa, muscularis propria, serosa and beyond, an increase in the proportion of high COX-2 positive carcinoma from $0 \%$ to $77 \%$ was observed with a decline in the low COX-2 positive tumors from T2 to T3. This association was statistically significant. A similar relation between depth of invasion and COX2 overexpression has been documented by Soumaoro (7) and $\mathrm{Wu}$ (17) in their studies. The number of high COX-2 positive tumors increased as the degree of tumor differentiation decreased. The proportion of high COX-2 expressing tumors increased from $56.2 \%$ to $66.6 \%$ and $100 \%$ in well differentiated, moderately differentiated and poorly differentiated carcinomas. Our findings are consistent with the studies of Masunaga (14) and Al-Maghrabi (11). We also observed that all of the mucinous and signet ring cell carcinomas showed high COX-2 positivity. These findings are in agreement with those by Baba et al. (18). A few others have described a relatively low COX-2 expression in signet ring cell carcinoma compared to the mucinous category (19). Comparing the COX-2 scores with the tumor size in the present study showed no statistically significant correlation similar to other previous studies $(7,14)$. Further comparing the total COX-2 scores of the primary tumor and the distant metastasis in the three stage IV tumors in the study, both the primary tumor and metastatic tumor were found to be high COX-2 expressors, raising the possibility for the role of COX-2 in tumor progression. However, more stage IV tumors need to be evaluated to assess the statistical significance of this correlation as there were only three stage IV tumors in the present study.

The association between COX-2 expression and tumor infiltrating lymphocytes (TIL) (CD8+) was not evaluated in the present study. Nosho et al. have reported that the number of TILs were increased in microsatellite instabile tumors but decreased in COX-2 expressing tumors (20).

In conclusion, COX-2 expression in colorectal carcinoma was investigated in the present study. The tumors were categorized as low positive and high positive based on their total COX-2 scores, which is the sum of proportion and intensity scores of staining. Left colonic carcinomas more frequently expressed COX-2 than the right colonic tumors. The association between COX-2 expression and depth of 
tumor invasion and the stage of tumor were statistically significant. The deeper the depth of invasion, the greater was the total COX-2 score. Additionally, the presence of lymph node metastasis increased (22.2\% to $25 \%$ to $47.7 \%)$ with increasing levels of COX-2 expression (negative to low positive to high positive) in the primary tumor. These findings may justify the use of selective COX-2 inhibitors such as Celecoxib, as an adjuvant to chemotherapy and radiotherapy in or combined usage of celecoxib with 5-fluorouracil which has been shown to improve chemosensitivity in chemorefractory colorectal carcinoma to improve recurrence free survival, disease free survival and overall survival $(21,22)$.

\section{CONFLICT of INTEREST}

The authors declare no conflict of interest.

\section{REFERENCES}

1. Cecilia M, Fenoglio P, Noffsinger AE, Stemmermann GN, Lantz PE, Isaacson PG. Gastrointestinal pathology. $3^{\text {rd }}$ ed. Philadelphia: Raven Press; 2008. 593-1016.

2. Ruder EH, Laiyemo AO, Graubard BI, Hollenbeck AR, Schatzkin A, Cross AJ. Non-steroidal anti-inflammatory drugs and colorectal cancer risk in a large, prospective cohort. Am J Gastroenterol. 2011;106:1340-50.

3. Friis S, Poulsen AH, Sørensen HT,Tjønneland A, Overvad K, Vogel U, McLaughlin JK, Blot WJ, Olsen JH. Aspirin and other non-steroidal anti-inflammatory drugs and risk of colorectal cancer: A Danish cohort study. Cancer Causes Control. 2009;20:731-40.

4. Din FV, Theodoratou E, Farrington SM, Tenesa A, Barnetson RA, Cetnarskyj R, Stark L, Porteous ME, Campbell H, Dunlop MG. Effect of aspirin and NSAIDs on risk and survival from colorectal cancer. Gut. 2010; 59:1670-9.

5. Hedin L, Rask K, Zhu Y, Wickman A, Wang W, Brevinge H, Thörn M, Ponten F, Sundfeldt K. Role of prostaglandins in colorectal tumorigenesis: Localization and expression of COX-1, COX-2, microsomal prostaglandin E synthase-1 and the EP2 receptor. Avicenna. 2013:5.

6. Jackson P, Blythe D. Immunohistochemical techniques. In: Bancroft JD, Gamble M (editors). Theory and practice of histological techniques. $6^{\text {th }}$ ed. London: Elsevier; 2008. 465.

7. Soumaoro LT, Uetake H, Higuchi T, Takagi Y, Enomoto M, Sugihara K. COX-2 expression: A significant prognostic indicator for patients with colorectal cancer. Clin Cancer Res. 2004;10:846571.

8. Tomozawa S, Tsuno NH, Sunami E, Hatano K, Kitayama J, Osada T. COX-2 expression correlates with tumor recurrence, especially hematogenous metastasis of colorectal cancer. $\mathrm{Br} \mathrm{J}$ Cancer. 2000;83: 324-8.

9. Greenhough A, Smartt HJ, Moore AE, Roberts HR, Williams AC, Paraskeva C, Kaidi A. The COX-2/PGE 2 pathway: Key roles in the hallmarks of cancer and adaptation to the tumor microenvironment. Carcinogenesis. 2009;30:377-86.
10. Tsujii M, Kawano S, Raymond N, Bois D. COX-2 expression in human colon and cancer cells increases metastatic potential. Proc Natl Acad Sci. 1997;94:3336-40.

11. Al-Maghrabi J, Buhmeida A, Emam E, Syrijanen K, Sibiany A, Al-Qahtani M , Al-Ahwal M. COX-2 expression as a predictors of outcome in colorectal carcinoma. World J Gastroenterol. 2012;18:1793-9.

12. Shehan KM, Sheehan K, Donoghue DP, Swuney FM, Conroy RM, Fitzgerald DJ, Murray FE. The relationship between COX-2 expression and colorectal cancer. JAMA. 1999; 282:1254-7.

13. Nasir A, Lopez A, Boulware D, Malafa M, Coppola D. Correlation between COX-2 and APC expression in left versus right-sided human colon cancer. Anticancer Res. 2011; 31:2191-5.

14. Masunaga R, Kohno H, Dhar DK. COX-2 expression correlates with tumor neovascularisation and prognosis in human colorectal carcinoma patients. Clin Cancer Res. 2000; 6:4064-8.

15. Fujita T, Matsui M, Takaku K, Uetake H, Ichikawa W, Taketo MM, Sugihara K. Size- and invasion-dependent increase in cyclooxygenase 2 levels in human colorectal carcinomas. Cancer Res. 1998;58:4823-6.

16. Elzagheid A, Emaetig F, Alkikhia L, Buhmeida A, Syrjänen K, ElFaitori O, Latto M, Collan Y, Pyrhönen S. High COX-2 expression is associated with advanced stages of colorectal cancer. Anticancer Res. 2013; 33:3137-43.

17. Wu AW, Gu J, Ji JF, Li ZF, Xu GW. Role of COX-2 in carcinogenesis of colorectal cancer and its relationship with tumor biological characteristics and patients prognosis. World J Gastroenterol. 2003;9:1990-4.

18. Baba Y, Nosho K, Shima K, Goessling W, Chan AT, Kimmie NG, Jeniffer AC, Edward LG, Charles SF, Ogino S. PTGER 2 overexpression in colorectal cancer is associated with microsatellite instability, independent of CpG island methylator phenotype. Cancer Epidemiol Biomarkers Prev. 2010;3:822-31.

19. Ogino S, Brahmandam M, Cantor M, Namgyal C, Kawasaki T, Kirkner G, Meyerhardt JA, Loda M, Fuchs CS. Distinct molecular features of colorectal carcinoma with signet ring cell component and colorectal carcinoma with mucinous component. Mod Pathol. 2006;19:59-68.

20. Nosho K, Baba Y, Tanaka N, Shima K, Hayashi M, Meyerhardt JA, Dranoff G, Fuchs CS, Ogino S. Tumor infiltrating T cell subsets, molecular changes in colorectal cancer and prognosis: Cohort study and literature review. J Pathol. 2010;222:350-66.

21. Rahman M, Selvarajan K, Hasan M, Chan AP, Jin C,Kim J, Chan Sk, Le ND, KimYB, Tai IT. Inhibition of COX-2 in colon cancer modulates tumor growth and MRD-1 Expression to enhance tumor regression in therapy-refractory cancers in Vivo. Neoplasia. 2012;14:624-33.

22. Ng K, Meyerhardt JA, Chan AT, Sato K, Chan JA, Niedzwiecki D, Saltz LB, Mayer RJ, Benson AB, Schaefer PL, Whittom R, Hantel A, Goldberg RM, Venook AP, Ogino S, Giovannucci EL, Fuchs CS. Aspirin and COX-2 inhibitor use in patients with stage III colon cancer. J Natl Cancer Inst. 2014;107:345. 